\title{
Síndrome de cola de caballo secundario a hernia discal
}

\author{
Santiago Sapriza*, Juan Manuel Velasco*, Nicolás Galli*, Leonardo Pereyra*, \\ Álvaro Rocchietti* , Fernando García*
}

\section{Resumen}

Introducción: el síndrome de cola de caballo (SCC) es una entidad poco frecuente, provocado por la compresión de las raíces nerviosas en el canal a nivel de la cola de caballo. Puede dejar graves secuelas si no es diagnosticado y tratado de forma precoz. Únicamente $2 \%$ a $6 \%$ de las hernias discales lumbares van a provocar un SCC. El diagnóstico de esta patología se basa en criterios clínicos, siendo éstos objeto de controversia dada la variabilidad de presentación del cuadro clínico. El objetivo de este trabajo es analizar la presentación clínica y evolución posoperatoria de los pacientes intervenidos por SCC secundaria a hernia de disco por equipo del Centro de Deformidades de Columna (CE.DEF.CO), entre enero de 2009 y diciembre de 2018.

Material y método: realizamos un análisis retrospectivo. La población objetivo son los pacientes intervenidos por SCC secundario a hernia discal entre enero de 2009 y diciembre de 2018 por equipo del CE.DEF.CO. Analizamos 20 pacientes intervenidos quirúrgicamente, de ellos $17(85 \%)$ casos presentaron síntomas urinarios, $18(90 \%)$ casos dolor o elementos deficitarios en miembros inferiores, 13 casos anestesia/hipoestesia en silla de montar, 6 casos síntomas intestinales y 3 casos presentaron disfunciones sexuales. En 19 casos se realizó procedimiento quirúrgico antes de las 48 de iniciados los síntomas.

Resultados: la remisión de síntomas esfinterianos al mes es de $83 \%$ y al año posoperatorio de casi $87 \%$. Se constató un caso de disfunción sexual al año posoperatorio.

Conclusión: el diagnóstico precoz por el médico emergencista es fundamental, por lo que el conocimiento de esta patología es imprescindible. Nuestros resultados en pacientes intervenidos de forma precoz, antes de las 48 horas, fueron similares a los publicados en la bibliografía internacional con bajo porcentaje de secuelas.

Palabras clave: Cauda equina

Síndrome cola de caballo

Hernia discal

Desplazamiento del disco intervertebral

Key words: $\quad$ Cauda equina

Cauda equina syndrome

Herniated disc

Intervertebral disc displacement

\footnotetext{
* Traumatólogo cirujano de columna. Centro de Deformidades de Columna (CE.DEF.CO). Aprobado por el Comité de ética en Investigación de CE.DEF.CO.

Correspondencia: Santiago Sapriza. Correo electrónico: santiagosapriza@hotmail.com.

Los autores declaran no tener conflicto de intereses.

Recibido: $11 / 5 / 20$

Aprobado: 23/9/20
} 


\section{Introducción}

El síndrome de cola de caballo o cauda equina (SCC) es una entidad poco frecuente, que configura una urgencia quirúrgica. La manifestación clínica es consecuencia de la compresión de las raíces nerviosas en el canal a nivel de la denominada cola de caballo. La región anatómica denominada cola de caballo es un grupo de raíces nerviosas que determina la sensación y funcionamiento de la vejiga, intestinos, órganos sexuales y miembros inferiores (figura 1).

La presentación clínica es variada, siendo fundamental su conocimiento para realizar un diagnóstico oportuno. La causa etiológica más frecuente es una hernia discal lumbar, siendo los niveles L4-L5 y L5-S1 los más frecuentemente afectados. Otras causas de SCC son los tumores espinales, hematomas, fracturas, procesos infecciosos, entre otras. Únicamente el $2 \%$ a $6 \%{ }^{(1)}$ de las hernias discales lumbares van a provocar un SCC. Se estima que la población general presenta una incidencia de 1:33.000 a 1:100.000, por lo que es una patología poco frecuente ${ }^{(2)}$.

El diagnóstico de SCC secundario a hernia discal se basa en criterios clínicos e imagenológicos. La presentación clínica ha sido un tema muy controversial, ya que la manifestación es muy variada en cuanto a la severidad de los síntomas, por lo que el diagnóstico lo realizamos cuando están presente uno o más de los siguientes sínto- mas: 1) alteración esfinteriana (urinario o intestinal), 2) hipoestesia en silla de montar o 3) disfunción sexual con posible déficit neurológico en miembros inferiores (motor, sensitivo y reflejos osteo-tendinosos) ${ }^{(3)}$.

Por tanto, cabe resaltar que un SCC se puede presentar sin dolor ni elementos neurológicos en miembros inferiores. Esto se debe a que la compresión se provoca por una hernia central, puede comprimir las raíces sacras y no necesariamente las raíces lumbosacras con localización más lateral $^{(4,5)}$.

Definimos el SCC como completo o incompleto según los criterios de Gleave y Macfarland, que se centran principalmente en el grado de compromiso esfinteriano ${ }^{(1)}$. Se define como incompleto aquel que se presenta con dificultades para orinar, alteración en la sensación al orinar, chorro débil y pérdida de deseo de movilizar intestino. Por otro lado, se define como completo aquel que se presenta con orina por rebozo, retención aguda de orina o incontinencia esfinteriana.

En cuanto a la imagenología, el gold standard es la resonancia magnética (RM). Las imágenes suelen ser muy evidentes, el fin de este estudio es descartar diferenciales y confirmar diagnóstico de hernia de disco y topografiar el nivel de compresión (figura 2).

La fisiopatología del SCC es discutida, siendo varias las vías patogénicas que se conjugan durante el proceso. La compresión mecánica pone en marcha una cascada

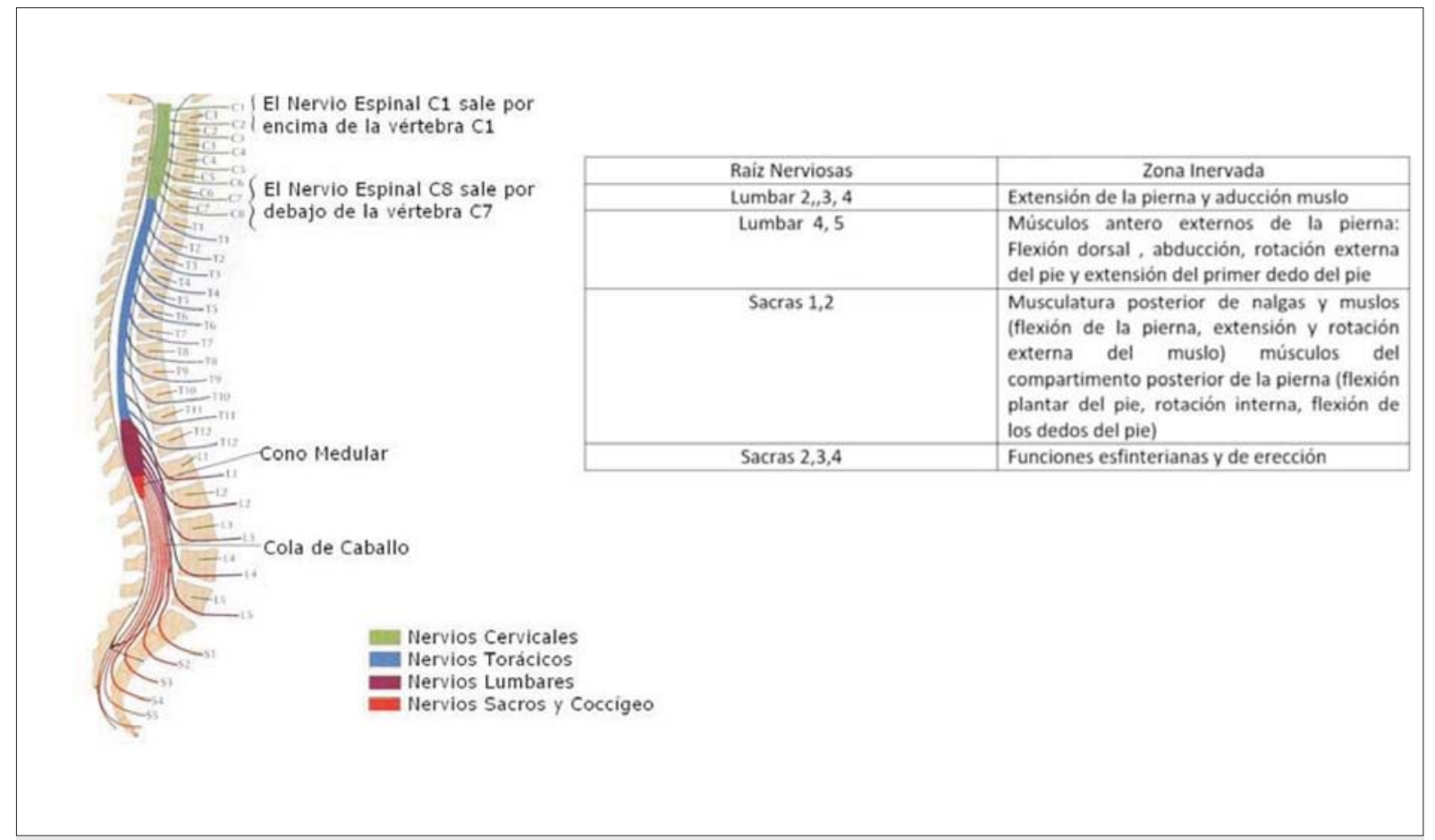

Figura 1. Recuerdo anatómico y funcional de cola de caballo. Tomado de:

http://fisio3unimetro.blogspot.com/2013/05/y-estructura-general-de-la-medula.html 


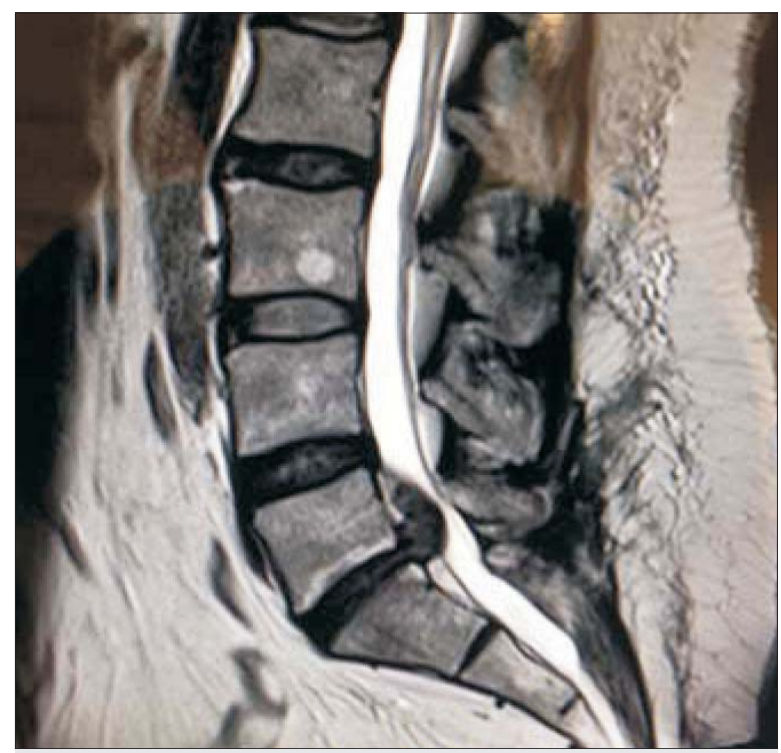

Figura 2. RM potenciada secuencia T2. Corte sagital. Voluminosa hernia de disco intervertebral extruida y migrada cefálica.

de alteraciones locales como la congestión venosa, la cascada inflamatoria a nivel conectivo y neuronal, cuya consecuencia final es la neuroisquemia (figura 3).

El SCC configura una urgencia quirúrgica. Sin embargo, existe controversia en la literatura acerca del momento adecuado para realizar la intervención, así como la relación entre mejoría del cuadro neurológico y momento de la cirugía ${ }^{(6,7)}$.

La mayoría de los autores coinciden en que la intervención quirúrgica precoz ofrece mejores resultados y la recomiendan dentro de las primeras 48 horas del inicio de los síntomas. La descompresión quirúrgica temprana podría revertir déficits neurológicos, prevenir pa- rálisis neurológicas irreversibles y reducir complicaciones como el dolor crónico ${ }^{(8)}$.

Este trabajo tiene como objetivo analizar la presentación clínica y evolución posoperatoria de los pacientes intervenidos por SCC secundaria a hernia de disco por equipo de CE.DEF.CO, entre enero de 2009 y diciembre de 2018.

\section{Material y método}

Realizamos un análisis retrospectivo de las características de los pacientes intervenidos por SCC secundario a hernia discal en los últimos diez años en nuestro servicio. La población objetivo son los pacientes intervenidos por SCC secundario a hernia discal entre enero de 2009 y diciembre de 2018. La recolección de datos la realizamos a partir de la historia clínica. Entendemos como SCC aquella compresión por hernia de disco a nivel de la cola de caballo que cumpla con los criterios clínicos previamente descriptos. Los clasificamos en completo e incompleto según criterios de Gleave y Macfarland. Registramos: edad, sexo, nivel de compresión, signo-sintomatología, tiempo entre cirugía y sintomatología y procedimiento realizado. El nivel de compresión lo obtuvimos de la imagenología, así como de la descripción operatoria. Entendemos por nivel a la unidad funcional comprometida, los dos cuerpos adyacentes y el disco intervertebral afectado. La variable signo-sintomatología se clasificó en cuatro variables: 1) alteración esfinteriana (urinario o intestinal), que se subclasificó como completa e incompleta; 2) hipoestesia en silla de montar, se subclasificó en presente/ausente; 3) disfunción sexual, que se subclasificó en disfunción eréctil, priapismo, impotencia sexual, disfunción eyaculatoria y pérdida de sensibilidad durante relaciones sexuales; 4) déficit neurológico en miembros inferiores (motor, sensitivo); se subclasificó en presente y ausente. La

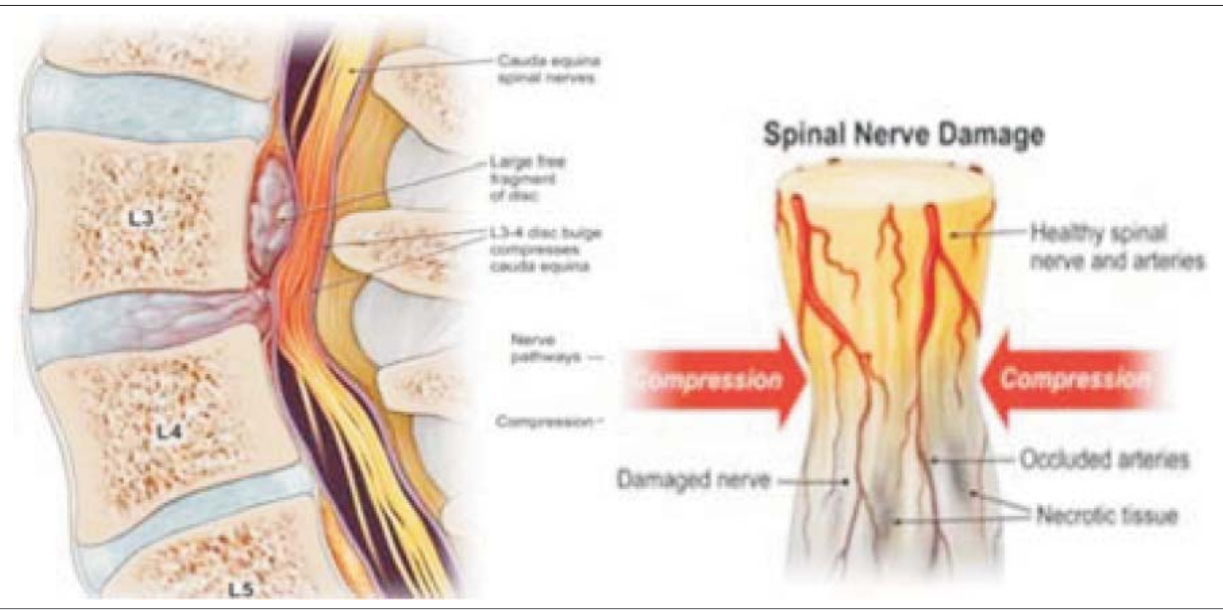

Figura 3. Patogenia de la neuroisquemia compresiva. 
presencia de déficit neurológico se clasificó en leve, moderado o severo. El procedimiento realizado lo clasificamos en dos variables: disectomía simple y liberación y artrodesis posterior instrumentada. El tiempo entre síntomas y cirugía se clasificó en tres variables: 1) antes de las 24 horas, 2) entre 24 y 48 horas de iniciados los síntomas y 3 ) después de las 48 horas de iniciados los síntomas.

Los criterios de exclusión fueron: historia clínica insuficiente y mínimo un año de seguimiento. Todos los casos fueron intervenidos y seguidos por equipo de cirujanos del Centro de Deformidades de Columna (CE.DEF.CO).

\section{Resultados}

Analizamos las historias clínicas de 21 pacientes intervenidos por SCC secundario a hernia de disco entre enero de 2009 y diciembre de 2019. De estos, un caso presentaba historia clínica incompleta, por lo que se excluyó. Del total de 20 casos, la media de edad fue 51 años (rango 28-72), de los cuales 14 (70\%) fueron hombres. El nivel más frecuentemente comprometido fue L4-L5 con 11 casos (55\%), seguido de L5-S1 con 6 casos, L3-L4 con 2 casos y por último L2-L3 con 1 caso. En cuanto al procedimiento realizado, 14 (70\%) pacientes fueron sometidos a disectomía simple y 6 a liberación y artrodesis posterior instrumentada con tornillos pediculares. En cuanto a la presentación clínica, 17 (85\%) casos presentaron síntomas urinarios, 18 (90\%) casos dolor o elementos deficitarios en miembros inferiores. De estos últimos, tres presentaron paresia de L5 severa y uno de raíz S1. De los 18, 17 casos presentaban dolor. Del total, 13 (65\%) casos anestesia/hipoestesia en silla de montar, 6 casos síntomas intestinales, y, por último, 3 casos presentaron disfunciones sexuales, uno de ellos con priapismo. Quince casos se presentaron como SCC incompletos, mientras los cinco restantes como completos. En cinco casos se realizó el procedimiento quirúrgico antes de las 24 horas de iniciados los síntomas, en 14 casos entre las 24 y 48 horas y en un caso luego de las 48 horas.

El resultado posoperatorio mostró que tres pacientes persistieron con alteraciones urinarias al mes posoperatorio, uno con incontinencia y dos con síntomas muy leves caracterizados por alteraciones funcionales documentadas por estudios de urodinamia. De los pacientes con dolor/déficit neurológico, en dos casos persistió paresia leve de L5 izquierda y en tres casos dolor lumbar mecánico. De los 13 pacientes que presentaron anestesia/hipoestesia en silla de montar al diagnóstico, 11 casos mostraron recuperación completa, persistiendo leve hiposetesia en dos casos. De los pacientes que presentaron disfunciones sexuales, uno persistió con síntomas.
De los seis pacientes que mostraron alteraciones intestinales solo en uno persistió sintomatología al año. Cabe destacar que un paciente de los que presentó SCC completo, persistió con síntomas: incontinencia urinaria, sensación de ampolla rectal llena e incontinencia y dolor lumbar. En tres casos se registró rotura del saco dural durante la cirugía, que fue reparado sin complicaciones en la evolución. No se registraron otras complicaciones intra ni posoperatorias.

\section{Discusión}

El SCC provocado por hernia de disco intervertebral es una entidad poco frecuente, que no supera el $2 \%$ de las hernias de disco que requieren cirugía. Por tanto, es difícil sacar conclusiones estadísticamente significativas en series que no superan los 40 casos. Por otro lado, contamos con pocos metaanálisis y revisiones sistemáticas. Nuestro centro tiene la ventaja de contar con historias clínicas completas y bien documentadas, con experiencia de 40 años en el tratamiento exclusivo de la patología de columna. Nuestra serie se extiende por un período de 10 años, comprende 20 casos, que si bien sabemos que estadísticamente no nos permite sacar conclusiones válidas, creemos que es representativa en nuestro país y nos permite realizar ciertos análisis.

Una de las controversias alrededor de esta entidad es la definición en cuanto al diagnóstico. En un artículo de revisión de literatura de Fraser y colaboradores, encontraron más de 17 definiciones del SCC, donde la mayoría de los autores la clasifica según la sintomatología ${ }^{(9,10)}$.

En nuestra clínica, seguimos los criterios diagnósticos propuestos por Fraser, en los que se hace diagnóstico con la imagenología y uno de los tres síntomas: 1) alteración esfinteriana (urinario o intestinal), 2) hipoestesia en silla de montar, o 3) disfunción sexual. El compromiso motor o sensitivo, al igual que el dolor ciático o crural bilateral, es frecuente, pero por sí solo no configura diagnóstico de SCC. Es importante establecer criterios diagnósticos para unificar conceptos con el objetivo de realizar un diagnóstico precoz. El retardo en el diagnóstico esta descripto en varios artículos y es uno de los factores que inciden en el pronóstico funcional ${ }^{(11,12)}$. En nuestra serie, la presentación del cuadro clínico es similar a las publicadas ${ }^{(1,6)}$, donde los síntoma más frecuentes son alteraciones urinarias y dolor, superando el $85 \%$ de los casos, seguidos de alteraciones sensitivas en silla de montar. En menor incidencia se encuentran las alteraciones intestinales ${ }^{(13)}$ y las disfunciones sexuales ${ }^{(4,14)}$.

Si bien nuestra serie se correlaciona con estos trabajos con mayor cantidad de casos y con el metaánalisis antes mencionado, creemos que las alteraciones sexuales e intestinales están subdiagnosticadas. Se trata se síntomas que son tan importantes como los urinarios, pero 


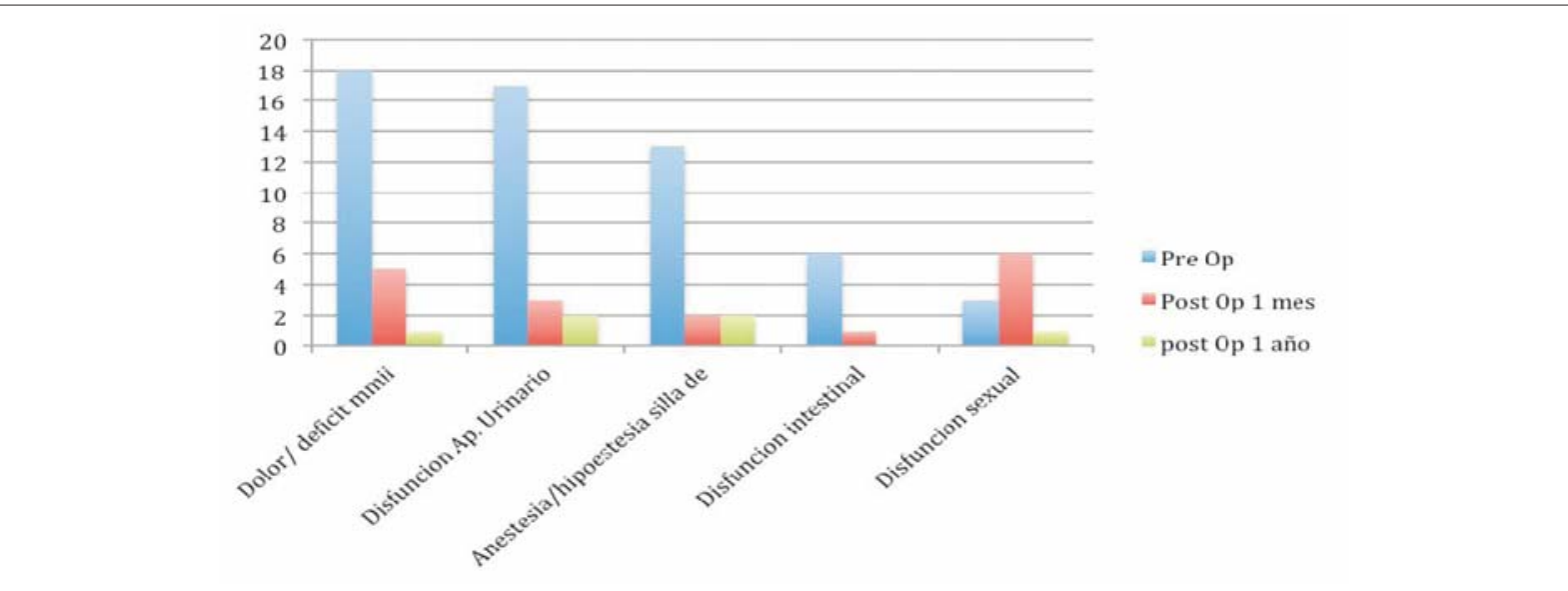

Figura 4.

que no son tan evidentes clínicamente y que el médico debe estar entrenado para realizar un interrogatorio y examen físico dirigido. Dentro de las disfunciones sexuales, se debe interrogar disfunción eréctil, priapismo, impotencia sexual, disfunción eyaculatoria y pérdida de sensibilidad durante relaciones sexuales. La mayoría de estos síntomas son difícil de constatar en el preoperatorio, en gran medida porque el cuadro es agudo y pasa desapercibido, y en gran parte porque el paciente es reticente a manifestar dichos síntomas. Sin embargo, en la evolución posoperatoria suele aparecer en el relato del paciente alguno de estos síntomas, por lo que creemos que están infradiagnosticados. Si bien tres casos mostraron disfunción sexual caracterizada por hipoestesia en genitales en dos casos y priapismo en otro, en el posoperatorio, además de estos tres, otros tres pacientes más refirieron algún grado de afectación en el posoperatorio mediato. En todos los casos la sintomatología revirtió completamente antes del año posoperatorio, excepto por un caso que presentó disfunción eréctil.

En cuanto a la recuperación de síntomas urinarios, el más preponderante por frecuencia, tiene una tasa de recuperación muy buena. En nuestra serie de los 18 pacientes que debutaron con síntomas urinarios solo uno persistió con sintomatología al año (figura 4). Cuatro pacientes requirieron interconsulta con urólogo para ser valorados y tratados por algún grado de disfunción transitoria.

Otra controversia en cuanto al SCC es la correlación entre síntomas y resultados clínicos posoperatorios. Hay trabajos que no mostraron diferencias entre la intervención realizada antes o después de las 48 horas. Por otro lado, uno de los metaanálisis más citados de Ahn y colaboradores $^{(15)}$, en 332 pacientes, al igual que otros trabajos $^{(16)}$, demostraron que aquellos intervenidos antes de las 48 horas mostraron mayor mejoría en síntomas uri- narios y rectales, además de elementos deficitarios. Incluso algunos autores ${ }^{(6,17)}$ mostraron que cuanto antes se realice la cirugía, mayor es la tasa de recuperación y menores las secuelas. También demostraron que aquellos con presentación completa tienen peor pronóstico funcional y se benefician de una cirugía precoz. En nuestra serie, el 70\% se realizó el procedimiento quirúrgico antes de las 48 horas de iniciados los síntomas, en $25 \%$ antes de las 24 horas y en un caso luego de las 48 horas. Por lo que no podemos extender conclusiones en cuanto al tiempo y evolución clínica. De todas maneras, notamos que las secuelas son menores a otras series publicadas. Creemos que esto se debe a que la mayoría, 14 casos, se intervinieron antes de las 48 horas. Un caso de la serie de presentación completa debutó con incontinencia esfinteriana, anestesia en silla de montar y parasia L5 izquierda. En este caso que se realizó tratamiento antes de las 24 horas; sin embargo, al año persistieron síntomas esfinterianos y deficitarios significativos. Cabe resaltar que no es infrecuente que los pacientes con SCC lleguen al equipo de columna, ya con 24 o 48 horas de evolución. En nuestro servicio creemos firmemente que el SCC es una verdadera urgencia y debe ser tratado lo antes posible.

En la mayoría de los trabajos no se especifica qué procedimiento quirúrgico se realizó. Existe una gama amplia que incluye hemi/laminectomía, disectomía abierta y endoscópica, microdisectomía y fusión del segmento, entre otros. En nuestra serie, 14 pacientes fueron sometidos a disectomía simple convencional y seis casos a liberación $\mathrm{y}$ artrodesis posterior instrumentada (figuras 5 y 6 ).

Cuatro de los pacientes sometidos a artrodesis presentaban canal estrecho congénito con hernias voluminosas. Los otros dos eran pacientes con importantes elementos degenerativos del espacio comprometido, como se evidencia en la figura 6, con gran hernia de disco con 


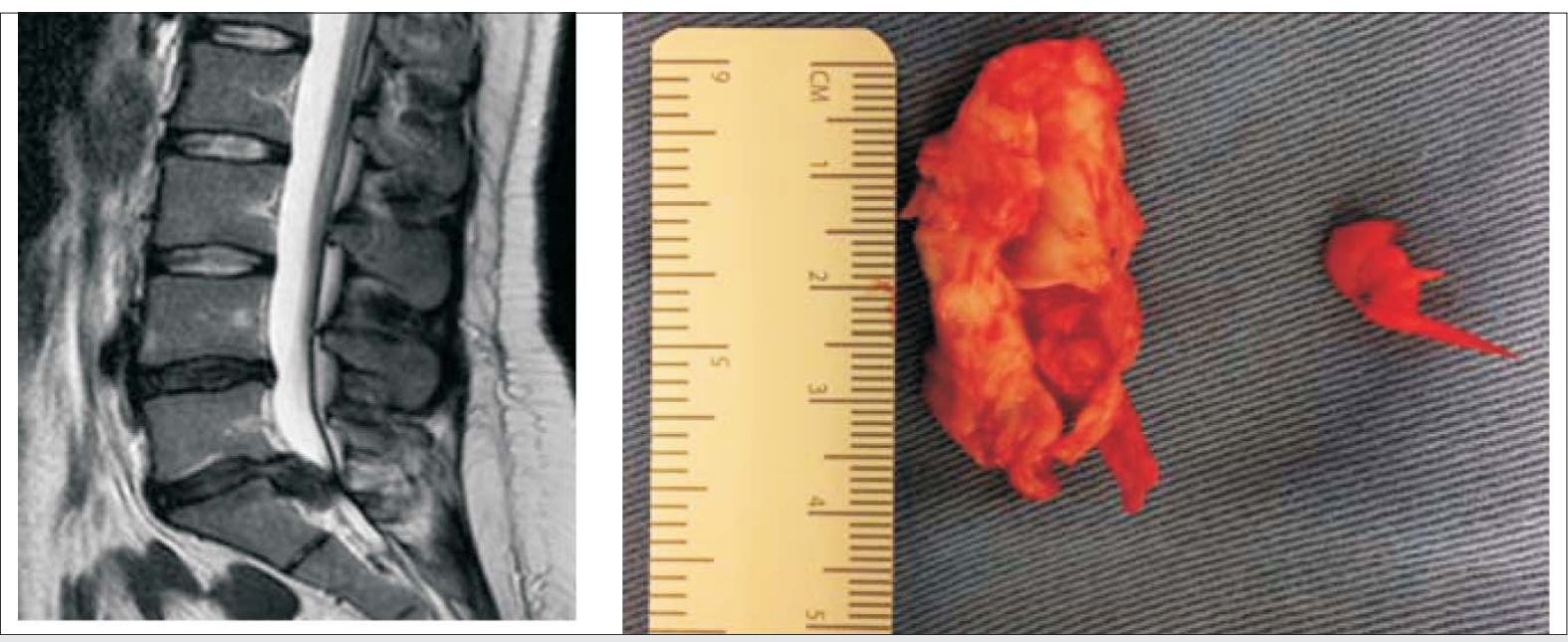

Figura 5. a) Resonancia magnética secuencia potenciada T2. Voluminosa hernia de disco L5-S1. b) Material herniario posoperatorio, disectomía simple.

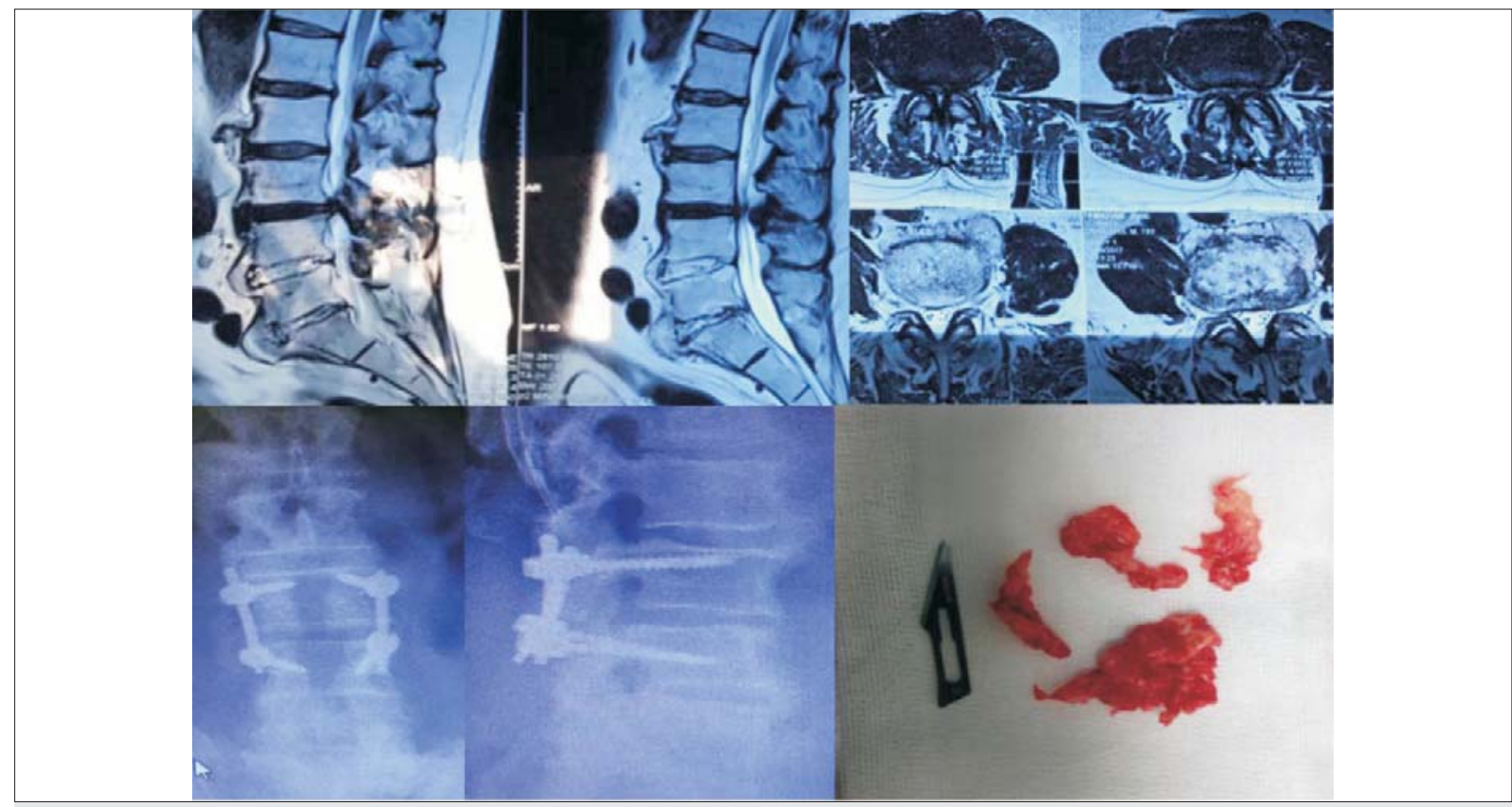

Figura 6. A y B. Resonancia magnética secuencia T2 sagital y axial, respectivamente. Obsérvese importantes elementos degenerativos a nivel L3-L4 que estenosan el canal descompensado con una hernia de disco. C) Radiografía posoperatoria. D) Material discal posoperatorio.

síntomas completos. En estos casos es conducta del servicio la instrumentación, ya que la disectomía simple tiene mayores riesgos de rotura del saco, pues el mismo suele estar friable y las raíces edematosas. Además, para lograr una liberación adecuada se requiere osteotomía de las articulares que pueden inestabilizar el segmento, por lo que suele requerir fijación con tornillos pediculares.
Las secuelas suelen ser importantes a nivel físico y psíquico. En general, se trata de pacientes jóvenes en los que la incontinencia esfinteriana, la disfunción sexual y el déficit motor son realmente complejos. Estos deben ser valorados con equipos multidisciplinarios con cirujano de columna, urólogo, psiquiatra, psicoterapia, etcétera. 


\section{Conclusión}

El SCC es una urgencia quirúrgica con secuelas graves a nivel esfinteriano, sexual y motor, si no se trata de forma adecuada.

El diagnóstico precoz por el médico emergencista es fundamental, ya que es el primer eslabón en la atención médica, por lo que el conocimiento de esta patología es imprescindible. Si bien nuestra serie de pacientes no nos permite obtener conclusiones estadísticamente significativas, nuestros resultados con el tratamiento quirúrgico precoz, antes de las 48 horas de iniciados los síntomas, se asemejan a los publicados en la bibliografía internacional con bajo porcentaje de secuelas.

\section{Summary}

Introduction: cauda equina syndrome (CES) is a rare entity, caused by compression of the nerve roots in the spinal canal at the cauda equina level. It can leave serious sequelae if it is not diagnosed and treated early. Only $2-6 \%$ of lumbar disc herniation will cause CES. The diagnosis of this pathology is based on clinical criteria, these being the subject of controversy given the variability of presentation of the clinical picture.

Materials and methods: we conducted a retrospective study. The target population are the patients operated on for CES secondary to herniated disc between January 2009 and December 2018 by a team from CE.DEF.CO. (center for spinal deformities). The objective is to carry out a set-up regarding the clinical presentation of this entity and to evaluate the correlation between surgical time and neurological improvement. Out of 20 patients who were operated, $17(85 \%)$ presented urinary symptoms, $18(90 \%)$ were patients in pain or presenting deficit elements in the lower limbs, 13 were cases of anesthesia / hypoesthesia in the saddle, 6 cases evidenced intestinal symptoms and 3 cases presented sexual dysfunction. In 19 cases, a surgical procedure was performed before 48 hours after symptoms started.

Results: the remission of sphincter symptoms after one month is $83 \%$ and it rises to almost $87 \%$ one year after the surgery. One case of sexual dysfunction persisted one year after surgery.

Conclusion: early diagnosis by the emergency physician is essential, so knowledge of this pathology is essential. Our experience and good results allow us to conclude that early surgical treatment is associated with symptomatic improvement and fewer neurological sequelae in the short and long term.

\section{Resumo}

Introdução: a síndrome da cauda eqüina (SCE) é uma entidade rara causada pela compressão das raízes nervosas no canal no nível da cauda eqüina.

Pode deixar seqüelas graves se não for diagnosticada e tratada precocemente. Apenas 2-6\% das hérnias do disco lombar causarão a síndrome da cauda eqüina. $\mathrm{O}$ diagnóstico desta patologia é baseado em critérios clínicos, sendo este motivo de controvérsia, dada a variabilidade da apresentação do quadro clínico.

Materiais e métodos: realizamos um estudo retrospectivo. A população-alvo estava composta por pacientes operados por SCE secundária a hérnia discal entre janeiro de 2009 e dezembro de 2018 pela equipe do CE. DEF.CO (Centro de Defeitos da Coluna Vertebral).

Foram analisados 20 pacientes operados, dos quais 17 (85\%) apresentaram sintomas urinários, 18 (90\%) dor ou elementos de deficiência em membros inferiores, 13 anestesia / hipoestesia em sela, 6 sintomas intestinais e 3 disfunções sexuais. Em 19 casos, um procedimento cirúrgico foi realizado antes de 48 horas após o início dos sintomas.

Resultado: a remissão dos sintomas esfincterianos em um mês é de $83 \%$ e em um ano pós-operatório é de quase $87 \%$. Um caso de disfunções sexuais foi encontrado um ano após a cirurgia.

Conclusão: o diagnóstico precoce pelo médico de emergência é essencial, portanto o conhecimento desta patologia é fundamental. Nossos resultados em pacientes operados precocemente, antes de 48 horas, foram semelhantes aos publicados na literatura internacional com baixo percentual de seqüelas.

\section{Bibliografía}

1. Gleave JRW, Macfarlane R. Cauda equina syndrome: what is the relationship between timing of surgery and outcome? Brit J Neurosurg 2002; 16(4):325-8.

2. Anthony S. Cauda equina syndrome. Med Protect Soc Casebook 2000; 20:9-13.

3. Quaile A. Cauda equina syndrome-the questions. Int Orthop 2019; 43(4):957-61

4. Kostuik JP, Harrington I, Alexander D, Rand W, Evans D. Cauda equina syndrome and lumbar disc herniation. J Bone Joint Surg Am 1986; 68:386-91.

5. Brown KL. Cauda equina syndrome. Implications for the orthopaedic nurse in a clinical setting. Orthop Nurs 1998; 17:31-5.

6. Kohles SS, Kohles DA, Karp AP, Erlich VM, Polissar NL. Time-dependent surgical outcomes following cauda equina syndrome diagnosis: comments on a meta-analysis. Spine (Phila Pa 1976) 2004; 29:1281-7.

7. Hoeritzauer I, Wood M, Copley PC, Demetriades AK, Woodfield $\mathbf{J}$. What is the incidence of cauda equina syndro- 
me? A systematic review. J Neurosurg Spine 2020; 1-10. doi: 10.3171/2019.12.SPINE19839.

8. Brouwers E, van de Meent H, Curt A, Starremans B, Hosman A, Bartels R. Definitions of traumatic conus medullaris and cauda equina syndrome: a systematic literature review. Spinal Cord 2017; 55(10):886-90.

9. Fraser S, Roberts L, Murphy E. Cauda equina syndrome: a literature review of its definition and clinical presentation. Arch Phys Med Rehabil 2009; 90:1964-8.

10. Mosdal C, Iversen P, Iversen-Hansen R. Bladder neuropathy in lumbar disc disease. Acta Neurochirur (Wien) 1979; 46:281-6.

11. Qureshi A, Sell P. Cauda equina syndrome treated by surgical decompression. Eur Spine J 2007; 16:2143-51.

12. Busse JW, Bhandari M, Schnittker JB, Reddy K, Dunlop RB. Delayed presentation of cauda equina syndrome secondary to lumbar disc herniation: functional outcomes and health-related quality of life. CJEM 2001; 3:285-91.
13. Tullberg T, Isacson J. Cauda equina syndrome with normal lumbar myelography. Acta Orthop Scand 1989; 60:265-7.

14. Mangialardi R, Mastorillo G, Minoia L, Garofalo R, Conserva F, Solarino GB. Lumbar disc herniation and cauda equina syndrome. Considerations on a pathology with different clinical manifestations. Chir Organi Mov 2002; 87:35-42.

15. Ahn UM, Ahn NU, Buchowski JM, Garrett ES, Sieber AN, Kostuik JP. Cauda equina syndrome secondary to lumbar disc herniation: a meta-analysis of surgical outcomes. Spine (Phila Pa 1976) 2000; 25:1515-22.

16. Shapiro S. Medical realities of cauda equina syndrome secondary to lumbar disc herniation. Spine (Phila Pa 1976) 2000; 25:348-52.

17. Todd NV. Cauda equina syndrome: the timing of surgery probably does influence outcome. Br J Neurosurg 2005; 19:301-6.

\section{Contribución de autores}

Cada autor contribuyó individual y significativamente para el desarrollo del manuscrito.

SS, NG, LP, JMV,AR y FG: revisión, realización de las cirugías y análisis de los datos.

SS, NG, LP y JMV: redacción, concepto intelectual, interpretación de resultados y diseño del proyecto.

Santiago Sapriza, https://orcid.org/0000-0003-1423-5540

Juan Manuel Velasco, http://orcid.org/0000-0001-8063-3091

Nicolás Galli, https://orcid.org/0000-0003-3830-5902

Leonardo Pereyra, http://orcid.org/0000-0002-8745-3058

Álvaro Rocchietti, http://orcid.org/0000-0001-5791-9665

Fernando García, https://orcid.org/0000-0001-6163-1402 